Moroccan J. of Pure and Appl. Anal. (MJPAA)

Volume 7(1), 2021, Pages 116-133

ISSN: Online 2351-8227 - Print 2605-6364

DOI: $10.2478 / \mathrm{mjpaa}-2021-0011$

\title{
Controlled K-Fusion Frame for Hilbert Spaces
}

\author{
NAdia ASSILA ${ }^{1}$, SAMir KABBAJ ${ }^{2}$, AND BRAhIM MOALIGE ${ }^{3}$
}

\begin{abstract}
Aвstract. K-fusion frames are a generalization of fusion frames in frame theory. In this paper, we extend the concept of controlled fusion frames to controlled $K$-fusion frames, and we develop some results on the controlled $K$-fusion frames for Hilbert spaces, which generalize some well known results of controlled fusion frame case. Also we discuss some characterizations of controlled Bessel $K$-fusion sequences and of controlled $K$-fusion frames. Further, we analyze stability conditions of controlled $K$-fusion frames under perturbation.
\end{abstract}

Mathematics Subject Classification (2020). 42C15, 46A35, 26A18.

Key words and phrases. Fusion Frame, K-Fusion Frame, Controlled Fusion Frame, Stability.

\section{Introduction}

Fames are more flexible than bases to solve some problems in Hilbert spaces. They were firstly introduced by Duffin and Schaeffer [11] to study nonharmonic Fourier series in 1952, and widely studied by Daubechies, Grossman and Meyer [10] in 1986. More results of frames are in [7].

Fusion frames as a genaralisation of frames were introduced by Casazza and Kutyniok in [6] and further there were developed in their joint paper [9] with Li. The theory for fusion frames

Received : July 07, 2020 - Accepted: November 18, 2020.

(C) The Author(s) 2020. This article is published with open access by Sidi Mohamed Ben Abdallah University.

1,2,3 Laboratory of Partial Differential Equations, Algebra and Spectral Geometry, Department of Mathematics, Faculty of Sciences, Ibn Tofail University, B.P. 133, Kenitra, Morocco

e-mail ${ }^{1}$ : nadia.assila@uit.ac.ma (Corresponding Author)

e-mail ${ }^{2}$ : samkabbaj@yahoo.fr

e-mail ${ }^{3}$ : brahim.moalige@uit.ac.ma. 
is available in arbitrary separable Hilbert spaces (finite-dimensional or not). The motivation behind fusion frames comes from signal processing, more precisely, the desire to process and analyze large data sets efficiently. A natural idea is to split such data sets into suitable smaller "blocks" which can be treated independently. From a pure mathematical point of view, fusion frames are special cases of the $g$-frames [22]. However, the connection to concrete applications is less apparent from the more abstract definition of $g$-frames. In 2012, L. Gavruta [15] introduced the notions of $K$-frames in Hilbert space to study the atomic systems with respect to a bounded linear operator $K$. Controlled frames in Hilbert spaces have been introduced by P. Balaz [1] to improve the numerical efficiency of iterative algorithms for inventing the frame operator. Further A. Khosravi [17] generalized this concept to the case of fusion frames. He has showed that controlled fusion framee as a generalization of fusion frames give a generalized way to obtain numerical advantage in the sense of preconditioning to check the fusion frame condition. In 2015 Rahimi [20] defined the concept of controlled $K$-frames in Hilbert spaces and showed that controlled $K$ - frames are equivalent to $K$-frames.

Motivated by the above literature, we introduce and investigate some properties of controlled $K$-fusion frames, we also generalize some known results for controlled fusion frames to controlled $K$-fusion frames. Finaly, we present perturbation result for controlled $K$-fusion frames. This paper is organized as follows. In Section 2, we recall several definitions about fusion frames, $K$-fusion frames and controlled fusion frames. Then, we give a basic properties about a bounded linear operator. In Section 3, we introduce the concept of controlled $K$-fusion frames and discuss their properties. In section 4 , we analyze stability conditions of controlled $K$-fusion frames under perturbation.

\section{Preliminaries and Notations}

Throughout this paper, we will adopt the following notations. $\mathcal{H}$ is a separable Hilbert space, $\left\{W_{i}\right\}_{i \in I}$ is a sequence of closed subspaces of $\mathcal{H}$, where $I$ is a countable index set. the family of all bounded linear operators on $\mathcal{H}$ is denoted by $B(\mathcal{H})$. We denote $\mathcal{R}_{T}, \mathcal{N}_{T}$, range and null space of a bounded linear operator $T$, respectively. $G L(\mathcal{H})$ is the set of all bounded invertible operators on $\mathcal{H}$ with bounded inverse, and $G L(\mathcal{H})^{+}$denotes the set of all positive operators in $G L(\mathcal{H}) . \pi_{W_{i}}$ is the orthogonal projection from $\mathcal{H}$ into $W_{i}$, and $\left\{w_{i}\right\}_{i \in I}$ is a family of weights, i.e. $w_{i}>0$, for any $i \in I$.

The space $\left(\oplus_{i \in I} \mathcal{H}\right)_{l^{2}}$ which is defined by

$$
\left(\oplus_{i \in I} \mathcal{H}\right)_{l^{2}}=\left\{\left\{f_{i}\right\}_{i \in I}: f_{i} \in \mathcal{H}, i \in I, \sum_{i \in I}\left\|f_{i}\right\|^{2}<\infty\right\},
$$

with the inner product as

$$
\left\langle\left\{f_{i}\right\}_{i \in I},\left\{g_{i}\right\}_{i \in I}\right\rangle=\sum_{i \in I}\left\langle f_{i}, g_{i}\right\rangle_{\mathcal{H}}
$$

is a separable Hilbert space [17].

\subsection{Fusion frames.}


Definition 2.1. [9] Let $\left\{W_{i}\right\}_{i \in I}$ be a family of closed subspaces of a Hilbert space $\mathcal{H}$. Let $\left\{w_{i}\right\}_{i \in I}$ be a family of weights, the family $\mathcal{W}=\left\{W_{i}, w\right\}_{i \in I}$ is called a $K$-fusion frame for $\mathcal{H}$, if there exist positive constants $A \leq B<\infty$ such that

$$
A\|f\|^{2} \leq \sum_{i \in I} w_{i}^{2}\left\|\pi_{W_{i}} f\right\|^{2} \leq B\|f\|^{2}, f \in \mathcal{H} .
$$

$A$ and $B$ are called the lower and upper bounds of fusion frame, respectively.

If only the right inequality of (2.1) holds, we call the family $\left\{W_{i}, w_{i}\right\}_{i \in I}$ a fusion bessel sequence.

\subsection{K-fusion frames.}

Definition 2.2. [3] Let $K \in B(\mathcal{H})$, let $\left\{W_{i}\right\}_{i \in I}$ be a family of closed subspaces of a Hilbert space $\mathcal{H}$, and let $\left\{w_{i}\right\}_{i \in I}$ be a family of weights. Then the family $\mathcal{W}=\left\{W_{i}, w_{i}\right\}_{i \in I}$ is called a $K$-fusion frame for $\mathcal{H}$, if there exist positive constants $A \leq B<\infty$ such that

$$
A\left\|K^{*} f\right\|^{2} \leq \sum_{i \in I} w_{i}^{2}\left\|\pi_{W_{i}} f\right\|^{2} \leq B\|f\|^{2}, f \in \mathcal{H} .
$$

Where $K^{*}$ is the adjoint operator of $K$.

$A$ and $B$ are called the lower and upper bounds of $K$-fusion frame, respectively.

suppose that $\left\{W_{i}, w_{i}\right\}_{i \in I}$ is a fusion Bessel sequence for $\mathcal{H}$, then the synthesis operator of $\left\{W_{i}, w_{i}\right\}_{i \in I}$ is defined by $T_{W}: \quad\left(\sum_{i \in I} \oplus W_{i}\right)_{l^{2}} \longrightarrow \mathcal{H}$,

$$
T_{W}\left(\left\{f_{i}\right\}_{i \in I}\right)=\sum_{i \in I} w_{i} f_{i}, \quad\left\{f_{i}\right\}_{i \in I} \in\left(\sum_{i \in I} \oplus W_{i}\right)_{l^{2}}
$$

Where

$$
\left(\sum_{i \in I} \oplus W_{i}\right)_{l^{2}}=\left\{\left\{f_{i}\right\}_{i \in I}: f_{i} \in W_{i}, i \in I, \sum_{i \in I}\left\|f_{i}\right\|^{2}<\infty\right\}
$$

Its adjoint operator, which is called the analysis operator $T_{W}^{*}: \quad \mathcal{H} \longrightarrow\left(\sum_{i \in I} \oplus W_{i}\right)_{l^{2}}$, is defined by

$$
T_{W}^{*}(f)=\left\{w_{i} \pi_{W_{i}} f\right\}_{i \in I}, \quad f \in \mathcal{H} .
$$

And the $K$-fusion frame operator associated is $S_{W}: \mathcal{H} \longrightarrow \mathcal{H}$.

$$
S_{W}(f)=\sum_{i \in I} w_{i}^{2} \pi_{W_{i}} f, \quad f \in \mathcal{H}
$$

\subsection{Controlled fusion frame.}

Definition 2.3. [17] Let $\left\{W_{i}\right\}_{i \in I}$ be a family of closed subspaces of a Hilbert space $\mathcal{H}$, let $\left\{w_{i}\right\}_{i \in I}$ be a family of weights, and let $T, U \in G L(\mathcal{H})$. Then the family $\mathcal{W}=\left\{W_{i}, w\right\}_{i \in I}$ is called a $(T, U)$-controlled fusion frame for $\mathcal{H}$, if there exist positive constants $A \leq B<\infty$ such that

$$
A\|f\|^{2} \leq \sum_{i \in I} w_{i}^{2}\left\langle\pi_{W_{i}} T f, \pi_{W_{i}} U f\right\rangle \leq B\|f\|^{2}, f \in \mathcal{H} .
$$


$A$ and $B$ are called the lower and upper bounds of $(T, U)$-controlled fusion frame, respectively. For further information in $K$-fusion frame and controlled fusion frame theory we refer the reader to [3], [17] [18] and [9].

In theory of frames, often use the following theorem, which describes some properties of the adjoint operator.

Theorem 1. [7] Let $\mathcal{H}_{1}, \mathcal{H}_{2}$ be Hilbert spaces, and suppose that $U \in B\left(\mathcal{H}_{1}, \mathcal{H}_{2}\right)$. Then,

i) $U^{*} \in B\left(\mathcal{H}_{2}, \mathcal{H}_{1}\right)$ and $\left\|U^{*}\right\|=\|U\|$.

ii) $U$ is surjective if and only if $\exists A>0$ such that $\left\|U^{*} h\right\|_{\mathcal{H}_{2}} \geq A\|h\|_{\mathcal{H}_{1}}$.

It is well-Known that not all bounded operator $U$ on a Hilbert space $\mathcal{H}$ is invertible: an operator $U$ needs to be injective and surjective in order to be invertible. For doing this, one can use right-inverse operator. The following lemma shows that if an operator $U$ has closed range, there exists a "right-inverse operator" $U^{+}$in the following sense:

Lemma 2. [7] Let $\mathcal{H}_{1}, \mathcal{H}_{2}$ be Hilbert spaces, and $U \in B\left(\mathcal{H}_{1}, \mathcal{H}_{2}\right)$ be a closed range $\mathcal{R}_{U}$. Then there exists a bounded operator $U^{\dagger}: \quad \mathcal{H}_{2} \longrightarrow \mathcal{H}_{1}$ for which

$$
U U^{\dagger} x=x, \quad x \in \mathcal{R}_{U},
$$

and

$$
\left(U^{*}\right)^{\dagger}=\left(U^{\dagger}\right)^{*} .
$$

The operator $U^{+}$is called the Pseudo-inverse of $U$.

In the literature, one will ofen see the pseudo-inverse of an operator $U$ with closed range defined as the unique operator $U^{\dagger}$ satisfying that

$$
\mathcal{N}_{U^{+}}=\mathcal{R}_{U}^{\perp}, \quad U U^{\dagger} x=x, \quad x \in \mathcal{R}_{U} .
$$

The following lemma is necessary for our results.

Lemma 3. [14] Let $V \subseteq \mathcal{H}$ be a closed subspace, and $T$ be a linear bounded operator on $\mathcal{H}$. Then

$$
\pi_{V} T^{*}=\pi_{V} T^{*} \pi_{\overline{T V}} .
$$

If $T$ is a unitary (i.e. $T^{*} T=T T^{*}=I d_{\mathcal{H}}$, then

$$
\pi_{\overline{T V}} T=T \pi_{V}
$$

Proposition 2.1. [21] Let $T: \mathcal{H} \longrightarrow \mathcal{H}$ be a linear operator. Then the following condition are equivalent:

(1) There exist $m>0$ and $M<\infty$, such that $m I \leq T \leq M I$;

(2) $T$ is positive and there exist $m>0$ and $M<\infty$, such that $m\|f\|^{2} \leq\left\|T^{\frac{1}{2}} f\right\|^{2} \leq M\|f\|^{2}$ for all $f \in \mathcal{H}$;

(3) $T$ is positive and $T^{\frac{1}{2}} \in G L(\mathcal{H})$;

(4) There exists a self-adjoint operator $A \in G L(\mathcal{H})$, such that $A^{2}=T$;

(5) $T \in G L^{+}(\mathcal{H})$.

The following lemma will be used in the sequel. 
Lemma 4. [12] Let $\mathcal{F}, \mathcal{G}, \mathcal{H}$ be Hilbert spaces. Let $T \in B(\mathcal{F}, \mathcal{G})$ and $T^{\prime} \in B(\mathcal{H}, \mathcal{G})$ with $\overline{\mathcal{R}}_{T^{*}}$ be orthogonally complemented.

Then the following statements are equivalent:

i) $T^{\prime} T^{*} \leq \lambda T T^{*}$ for some $\lambda>0$.

ii) There exists $\mu>0$ such that $\left\|T^{*} z\right\| \leq \mu\left\|T^{*} z\right\|$, for all $z \in \mathcal{G}$.

Theorem 5. [13] Let $T$ be a positive linear bounded operator on $\mathcal{H}$. $T$ possesses a unique positive bounded square root which commutes with every bounded operator that commutes with $T$.

\section{Controlled $K$-fusion frame}

In this section, we introduce the notion of controlled $K$-fusion frames in Hilbert spaces and we discuss some their properties.

Definition 3.1. Let $K \in B(\mathcal{H})$, and $\left\{W_{i}\right\}_{i \in I}$ be a family of closed subspaces of a Hilbert space $\mathcal{H}$. Also, let $\left\{w_{i}\right\}_{i \in I}$ be a family of weights, and let $C, C^{\prime} \in G L(\mathcal{H})$. Then $\mathcal{W}=\left\{W_{i}, w_{i}\right\}_{i \in I}$ is called a $K$-fusion frame controlled by $C$ and $C^{\prime}$ or $\left(C, C^{\prime}\right)$-controlled $K$-fusion frame if there exist two constantants

$$
0<A_{C C^{\prime}} \leq B_{C C^{\prime}}<\infty
$$

such that

$$
A_{C C^{\prime}}\left\|K^{*} f\right\|^{2} \leq \sum_{i \in I} w_{i}^{2}<\pi_{W_{i}} C f, \pi_{W_{i}} C^{\prime} f>\leq B_{C C^{\prime}}\|f\|^{2}, f \in \mathcal{H} .
$$

Where $K^{*}$ is the adjoint operator of $K$.

$A_{C C^{\prime}}$ and $B_{C C^{\prime}}$ are called lower and upper bounds of a $\left(C, C^{\prime}\right)$-controlled $K$-fusion frame respectively.

(1) We call $\mathcal{W}$ a $\left(C, C^{\prime}\right)$-controlled Parsval $K$-fusion frame if $A_{C C^{\prime}}=B_{C C^{\prime}}=1$.

(2) If only the second inequality (3.1) is required, we call $\mathcal{W}$ a $\left(C, C^{\prime}\right)$-controlled Bessel $K$-fusion sequence with Bessel bound $B$.

Remark 6. i) If $K=I$ ( where is the identity operator), then every $\left(C, C^{\prime}\right)$-controlled $K$-fusion frame is a $\left(C, C^{\prime}\right)$-controlled fusion frame.

ii) If $C=C^{\prime}=I$, then every $\left(C, C^{\prime}\right)$-controlled $K$-fusion frame is a K-fusion frame.

iii) Every $\left(C, C^{\prime}\right)$-controlled fusion frame is a $\left(C, C^{\prime}\right)$-controlled $K$-fusion frame. Indeed, by definition (3.1) there exist constants $0<A_{C C^{\prime}} \leq B_{C C^{\prime}}$, such that for all $f \in \mathcal{H}$, we have

$$
A_{C C^{\prime}}\|f\|^{2} \leq \sum_{i \in I} w_{i}^{2}<\pi_{W_{i}} C f, \pi_{W_{i}} C^{\prime} f>\leq B_{C C^{\prime}}\|f\|^{2} .
$$

Therefore, for $\|K\|>0$, one has

$$
A_{C C^{\prime}}\left\|K^{*} f\right\|^{2} \leq A_{C C^{\prime}}\left\|K^{*}\right\|^{2}\|f\|^{2} \leq A_{C C^{\prime}}\|K\|^{2}\|f\|^{2},
$$

that is,

$$
\frac{A_{C C^{\prime}}}{\|K\|^{2}}\left\|K^{*} f\right\|^{2} \leq A_{C C^{\prime}}\|f\|^{2},
$$


it follows that,

$$
\frac{A_{C C^{\prime}}}{\|K\|^{2}}\left\|K^{*} f\right\|^{2} \leq \sum_{i \in I} w_{i}^{2}<\pi_{W_{i}} C f, \pi_{W_{i}} C^{\prime} f>\leq B_{C C^{\prime}}\|f\|^{2} .
$$

Hence, the family $\mathcal{W}$ is a $\left(C, C^{\prime}\right)$-controlled $K$-fusion frame for $\mathcal{H}$.

The next example shows that in general, frames may be controlled $K$-fusion frame without being a controlled fusion frame.

Example 3.1. Let $\mathcal{H}=l_{2}(\mathbb{C})=\left\{\left.\left\{a_{n}\right\}_{n \in \mathbb{N}} \subset \mathbb{C}\left|\sum_{n=0}^{+\infty}\right| a_{n}\right|^{2}<\infty\right\}$ be a Hilbert space, with respect to the inner product

$$
\left\langle\left\{a_{n}\right\}_{n \in \mathbb{N}},\left\{b_{n}\right\}_{n \in \mathbb{N}}\right\rangle=\sum_{n \in \mathbb{N}} a_{n} \bar{b}_{n}
$$

equipped with the norm

$$
\left\|\left\{a_{n}\right\}_{n \in \mathbb{N}}\right\|_{l_{2}(\mathbb{C})}=\left(\sum_{n \in \mathbb{N}}\left|a_{n}\right|^{2}\right)^{\frac{1}{2}} .
$$

Consider two operators $C$ and $C^{\prime}$ defined by

$$
\begin{aligned}
C: \mathcal{H} & \longrightarrow \mathcal{H} \\
\left\{a_{n}\right\}_{n \in \mathbb{N}} & \longmapsto\left\{\alpha a_{n}\right\}_{n \in \mathbb{N}} \\
\text { resp. } C^{\prime}: \mathcal{H} & \longrightarrow \mathcal{H} \\
\left\{a_{n}\right\}_{n \in \mathbb{N}} & \longmapsto\left\{\beta a_{n}\right\}_{n \in \mathbb{N}}
\end{aligned}
$$

where $\alpha, \beta \in \mathbb{R}_{+}^{*}$.

It is easy to see that:

- $C$ and $C^{\prime}$ are positives.

- $C$ and $C^{\prime}$ are invertibles.

Then, invertible operators are given respectively by:

$$
\begin{aligned}
C^{-1}: \mathcal{H} & \longrightarrow \mathcal{H} \\
\left\{a_{n}\right\}_{n \in \mathbb{N}} & \longmapsto\left\{\alpha^{-1} a_{n}\right\}_{n \in \mathbb{N}},
\end{aligned}
$$

and

$$
\begin{aligned}
C^{\prime-1}: \mathcal{H} & \longrightarrow \mathcal{H} \\
\left\{a_{n}\right\}_{n \in \mathbb{N}} & \longmapsto\left\{\beta^{-1} a_{n}\right\}_{n \in \mathbb{N}} .
\end{aligned}
$$

Let $E_{i}=\left\{a_{j}\right\}_{j \in \mathbb{N}}$, where $a_{j}=\left\{\delta_{i}^{j}\right\}_{i \in \mathbb{N}}$ (where $\delta_{i}^{j}$ is the Kronecker symbol). Let $\left\{W_{i}\right\}_{i \in \mathbb{N}}$ be a closed subspaces of $\mathcal{H}$ such that $W_{i}=\mathbb{C} E_{i}$, and let $w_{i}=\frac{1}{\sqrt{i+1}}$, for all $i \in \mathbb{N}$.

The family $\mathcal{W}=\left\{W_{i}, w_{i}\right\}_{i \in \mathbb{N}}$ is a $\left(C, C^{\prime}\right)$-controlled Bessel fusion sequence. Indeed for each $\left\{a_{n}\right\}_{n \in \mathbb{N}} \in$ $\mathcal{H}$, we have

$$
\sum_{i \in \mathbb{N}} w_{i}^{2}\left\langle\pi_{W_{i}} C\left(\left\{a_{n}\right\}_{n \in \mathbb{N}}\right), \pi_{W_{i}} C^{\prime}\left(\left\{a_{n}\right\}_{n \in \mathbb{N}}\right)\right\rangle=\alpha \beta \sum_{i \in \mathbb{N}} \frac{1}{i+1}\left|a_{i}\right|^{2}
$$




$$
\begin{aligned}
& \leq \alpha \beta \sum_{i \in \mathbb{N}}\left|a_{i}\right|^{2} \\
& \left.=\alpha \beta \|\left\{a_{n}\right\}_{n \in \mathbb{N}}\right) \|_{\mathcal{H}}^{2} .
\end{aligned}
$$

But is not $\left(C, C^{\prime}\right)$-controlled fusion frame, For this, assume the contrary that exists $A_{C C^{\prime}}>0$ such that:

$$
A_{C C^{\prime}} \sum_{i \in \mathbb{N}}\left|a_{i}\right|^{2} \leq \sum_{i \in \mathbb{N}} \frac{\alpha \beta}{i+1}\left|a_{i}\right|^{2} \text {. }
$$

Hence

$$
\sum_{i \in \mathbb{N}}\left|a_{i}\right|^{2}<\infty \Longrightarrow \lim _{i \longrightarrow+\infty} a_{i}=0
$$

So, we have

$$
\begin{gathered}
\left|a_{j}\right| \longrightarrow 0 \text { as } \quad \longrightarrow \longrightarrow \infty ; \\
\frac{\alpha \beta}{i+1} \longrightarrow 0 \text { as } i \longrightarrow \infty . \\
\Longrightarrow\left\{\begin{array}{c}
\forall \varepsilon \geq 0 \quad \exists N \in \mathbb{N}: \quad j \geq N \Longrightarrow\left|a_{j}\right|<\varepsilon, \\
\forall \gamma \geq 0 \quad \exists N \in \mathbb{N}: \quad j \geq M \Longrightarrow \frac{\alpha \beta}{j+1}<\gamma .
\end{array}\right. \\
\Longrightarrow \sum_{i \in \mathbb{N}} \frac{\alpha \beta-(j+1) A_{C C^{\prime}}}{j+1}\left|a_{j}\right|^{2} \geq 0 .
\end{gathered}
$$

By fixing $\varepsilon=\gamma$, there exist $N, M \in \mathbb{N}^{*}$, such that

$$
\left\{\begin{array}{l}
j \geq N \quad \Longrightarrow\left|a_{j}\right|<\varepsilon, \\
j \geq M \quad \Longrightarrow \frac{\alpha \beta}{j+1}<\varepsilon .
\end{array}\right.
$$

Now, let $N_{1}=\max (N, M)$, then $\forall j \geq N_{1},\left|a_{j}\right|<\varepsilon$ and $\frac{\alpha \beta}{i+1}<\varepsilon$. Hence

$$
\sum_{i=0}^{N_{1}-1} \frac{\alpha \beta-(i+1) A_{C C^{\prime}}}{i+1}\left|a_{i}\right|^{2}+\varepsilon^{2} \sum_{i=N_{1}}^{\infty}\left(\varepsilon-A_{C C^{\prime}}\right) \geq 0 .
$$

Now, for $\varepsilon=\frac{A_{C C^{\prime}}}{2}$, we obtain

$$
\sum_{i=0}^{N_{1}-1} \frac{\alpha \beta-(i+1) A_{C C^{\prime}}}{i+1}\left|a_{i}\right|^{2}+\left(\frac{A_{C C^{\prime}}}{2}\right)^{2} \sum_{i=N_{1}}^{\infty}\left(\frac{-A_{C C^{\prime}}}{2}\right) \geq 0 .
$$

absurde.

Now if we considere the operator

$$
\begin{aligned}
K: \mathcal{H} & \longrightarrow \mathcal{H} \\
\left\{a_{n}\right\}_{n \in \mathbb{N}} & \longmapsto\left\{\frac{a_{n}}{\sqrt{n+1}}\right\}_{n \in \mathbb{N}} .
\end{aligned}
$$


Then, $K$ is a bounded linear operator on $\mathcal{H}$. Furthermore, for each $\left\{a_{n}\right\}_{n \in \mathbb{N}} \in \mathcal{H}$, we have

$$
\left\langle K^{*}\left(\left\{a_{n}\right\}_{n \in \mathbb{N}}\right), K^{*}\left(\left\{a_{n}\right\}_{n \in \mathbb{N}}\right)\right\rangle=\sum_{i=0}^{\infty} \frac{\left|a_{n}\right|^{2}}{n+1} .
$$

So,

$$
\sum_{i=0}^{\infty} \frac{\alpha \beta}{2} \frac{\left|a_{n}\right|^{2}}{n+1} \leq \sum_{i=0}^{\infty} \frac{\alpha \beta}{n+1}\left|a_{n}\right|^{2} \leq \alpha \beta \sum_{i=0}^{\infty}\left|a_{n}\right|^{2},
$$

The following proposition provides a relation between controlled $K$-fusion frames and controlled fusion frames.

Proposition 7. Let $K \in \mathcal{B}(\mathcal{H})$ be a closed range operator. Then, every $\left(C, C^{\prime}\right)$-controlled $K$-fusion frame is a $\left(C, C^{\prime}\right)$-controlled fusion frame for $\mathcal{R}_{K}$.

Proof. Let $\mathcal{W}=\left\{\left(W_{i}, w_{i}\right)\right\}_{i \in I}$ be a $\left(C, C^{\prime}\right)$-controlled $K$-fusion frame with frame bounds $A_{C C^{\prime}}$ and $B_{C C^{\prime}}$. Then for all $f \in \mathcal{R}_{K}$, we have

$$
A_{C C^{\prime}}\left\|K^{*} f\right\|^{2} \leq \sum_{i \in I} w_{i}^{2}<\pi_{W_{i}} C f, \pi_{W_{i}} C^{\prime} f>\leq B_{C C^{\prime}}\|f\|^{2} .
$$

Therefore, via lemma 2, we have

$$
A_{C C^{\prime}}\|f\|^{2} \leq A_{C C^{\prime}}\left\|\left(K^{*}\right)^{\dagger} f\right\|^{2}\left\|K^{*} f\right\|^{2} .
$$

Hence,

$$
\frac{A_{C C^{\prime}}}{\left\|\left(K^{*}\right)^{\dagger}\right\|^{2}}\|f\|^{2} \leq A_{C C^{\prime}}\left\|K^{*} f\right\|^{2}
$$

Thus,

$$
\frac{A_{C C^{\prime}}}{\left\|\left(K^{*}\right)^{\dagger}\right\|^{2}}\|f\|^{2} \leq \sum_{i \in I} w_{i}^{2}<\pi_{W_{i}} C f, \pi_{W_{i}} C^{\prime} f>\leq B_{C C^{\prime}}\|f\|^{2} .
$$

So, we have the result.

If $\mathcal{W}$ is a $\left(C, C^{\prime}\right)$-controlled $K$-fusion frame and $C^{\prime *} \pi_{W_{i}} C$ is a positive operator for each $i \in I$, then $C^{* *} \pi_{W_{i}} C=C^{*} \pi_{W_{i}} C^{\prime}$ and we have

$$
A_{C C^{\prime}}\left\|K^{*} f\right\|^{2} \leq \sum_{i \in I} w_{i}^{2}\left\|\left(C^{\prime *} \pi_{W_{i}} C\right)^{\frac{1}{2}} f\right\|^{2} \leq B_{C C^{\prime}}\|f\|^{2}, f \in \mathcal{H} .
$$

Indeed,

$$
\begin{aligned}
\sum_{i \in I} w_{i}^{2}\left\langle\pi_{W_{i}} C f, \pi_{W_{i}} C^{\prime} f\right\rangle & =\sum_{i \in I} w_{i}^{2}\left\langle C^{\prime *} \pi_{W_{i}} C f, f\right\rangle \\
& =\sum_{i \in I} w_{i}^{2}\left\langle\left(C^{\prime *} \pi_{W_{i}} C\right)^{\frac{1}{2}} f,\left(C^{\prime *} \pi_{W_{i}} C\right)^{\frac{1}{2}} f\right\rangle \\
& =\sum_{i \in I} w_{i}^{2}\left\|\left(C^{*} \pi_{W_{i}} C\right)^{\frac{1}{2}} f\right\|^{2} .
\end{aligned}
$$

We define the controlled analysis operator by

$$
T_{C C^{\prime}}: \mathcal{H} \longrightarrow \mathcal{K}
$$




$$
f \longmapsto T_{C C^{\prime}}(f):=\left(w_{i}\left(C^{\prime *} \pi_{W_{i}} C\right)^{\frac{1}{2}} f\right)_{i \in I}
$$

Where

$$
\mathcal{K}=\left\{\left(w_{i}\left(C^{* *} \pi_{W_{i}} C\right)^{\frac{1}{2}} f\right)_{i \in I} \mid f \in \mathcal{H}\right\} \subseteq\left(\oplus_{i \in I} \mathcal{H}\right)_{l^{2}}
$$

$\mathcal{K}$ is closed [17] and $T_{C C^{\prime}}$ is well defined. Morever $T_{C C^{\prime}}$ is a bounded linear operator. Its adjoint operator is given by

$$
\begin{aligned}
T_{C C^{\prime}}^{*}: \mathcal{K} & \longrightarrow \mathcal{H} \\
\left.\left(w_{i}\left(C^{* *} \pi_{W_{i}} C\right)^{\frac{1}{2}} f\right)_{i \in I}\right) & \longmapsto T_{C C^{\prime}}^{*}\left(\left(w_{i}\left(C^{*} \pi_{W_{i}} C\right)^{\frac{1}{2}} f\right)_{i \in I}\right):=\sum_{i \in I} w_{i}^{2} C^{*} \pi_{W_{i}} C f,
\end{aligned}
$$

and is called the controlled synthesis operator.

Therefore, we define the controlled $K$-fusion frame operator $S_{C C^{\prime}}$ on $\mathcal{H}$ by

$$
S_{C C^{\prime}}=T_{C C^{\prime}}^{*} T_{C C^{\prime}}(f)=\sum_{i \in I} w_{i}^{2} C^{*} \pi_{W_{i}} C f, \quad f \in \mathcal{H}
$$

In fact, many of the properties of the ordinary $K$-fusion frames are valid in this case.

Lemma 8. Let $\mathcal{W}=\left\{W_{i}, w_{i}\right\}_{i \in I}$ be a $\left(C, C^{\prime}\right)$-controlled K-fusion frame with bounds $A_{C C^{\prime}}$ and $B_{C C^{\prime}}$. Then the operator $S_{C C^{\prime}}$ (3.3) is a well defined, linear, positive, bounded and self-adjoint operator. Furthermore, we have

$$
A_{C C^{\prime}} K K^{*} \leq S_{C C^{\prime}} \leq B_{C C^{\prime}} I d_{\mathcal{H}}
$$

Proof. $\quad$ By definition, $S_{C C^{\prime}}$ is a linear bounded and well defined operator, and it is clear to see that $S_{C C^{\prime}}$ is a positive and self-adjoint operator.

- The family $\mathcal{W}=\left\{W_{i}, w\right\}_{i \in I}$ is a $\left(C, C^{\prime}\right)$-controlled $K$-fusion frame for $\mathcal{H}$ with bounds $A_{C C^{\prime}}$ and $B_{C C^{\prime}}$ if and only if

$$
A_{C C^{\prime}}\left\|K^{*} f\right\|^{2} \leq\left\langle S_{C C^{\prime}} f, f\right\rangle=\left\langle\sum_{i \in I} w_{i}^{2} C^{\prime *} \pi_{W_{i}} C f, f\right\rangle \leq B_{C C^{\prime}}\|f\|^{2}, \quad f \in \mathcal{H},
$$

that is,

$$
A_{C C^{\prime}}\left\langle K K^{*} f, f\right\rangle \leq\left\langle S_{C C^{\prime}} f, f\right\rangle \leq B_{C C^{\prime}}\langle f, f\rangle, \quad f \in \mathcal{H}
$$

Hence,

$$
A_{C C^{\prime}} K K^{*} \leq S_{C C^{\prime}} \leq B_{C C^{\prime}} \cdot I d_{\mathcal{H}}
$$

so the conclusion holds.

The next theorem generalizes the situation of controlled Bessel $K$-fusion sequence. Since it has similar procedure, the proof is omitted.

Theorem 9. $\mathcal{W}$ is a $\left(C, C^{\prime}\right)$-controlled Bessel $K$-fusion sequence with bound $B_{C C^{\prime}}$ if and only if $T_{C C^{\prime}}^{*}$ is well-defined bounded operator and $\left\|T_{C C^{\prime}}^{*}\right\| \leq \sqrt{B}$. 
Controlled K-fusion frame operator of $\left(C, C^{\prime}\right)$-controlled $K$-fusion frame is not invertible in general, but we can show that it is invertible on the subspace $\mathcal{R}_{K} \subset \mathcal{H}$. In fact, since $\mathcal{R}_{K}$ is closed

$$
\left.K K^{\dagger}\right|_{\mathcal{R}_{K}}=i d_{\mathcal{R}_{K^{\prime}}}
$$

so we have

$$
i d_{\mathcal{R}_{K}}^{*}=\left(\left.K^{\dagger}\right|_{\mathcal{R}_{K}}\right)^{*} K^{*}
$$

Hence for any $f \in \mathcal{R}_{K}$

$$
\|f\|=\left\|\left(\left.K^{\dagger}\right|_{\mathcal{R}_{K}}\right)^{*} K^{*} f\right\| \leq\left\|K^{\dagger}\right\|\left\|K^{*} f\right\|,
$$

that is,

$$
\|f\|^{2} \leq\left\|K^{\dagger}\right\|^{2}\left\|K^{*} f\right\|^{2}
$$

Combined with (3.1) we have

$$
\left\langle S_{C C^{\prime}} f, f\right\rangle \geq A_{C C^{\prime}}\left\|K^{*} f\right\|^{2} \geq A_{C C^{\prime}}\left\|K^{\dagger}\right\|^{2}\|f\|^{2}, \quad \forall f \in \mathcal{R}_{K} .
$$

So from the definition of $\left(C, C^{\prime}\right)$-controlled $K$-fusion frame, one implies that $S: \quad \mathcal{R}_{K} \longrightarrow$ $S\left(\mathcal{R}_{K}\right)$ is an isomorphism, furthermore we have

$$
B_{C C^{\prime}}^{-1}\|f\| \leq\left\|S^{-1} f\right\| \leq A_{C C^{\prime}}^{-1}\left\|K^{\dagger}\right\|^{2}\|f\|, \forall f \in\left(S\left(\mathcal{R}_{K}\right)\right) .
$$

Theorem 10. Let $K \in B(\mathcal{H})$ be a closed range operator, then $\mathcal{W}$ is a $\left(C, C^{\prime}\right)$-controlled $K$-fusion frame with bounds $A_{C^{\prime} C^{\prime}}$ and $B_{C C^{\prime}}$ if and only if $T_{C C^{\prime}}^{*}$ is well-defined and surjective.

Proof. Let the sequence $\mathcal{W}$ be a $\left(C, C^{\prime}\right)$-controlled $K$-fusion frame for $\mathcal{H}$, and let $S_{C C^{\prime}}$ be its controlled $K$-fusion frame operator. Then, it is a $\left(C, C^{\prime}\right)$-controlled Bessel $K$-fusion sequence and therefore, by Theorem 9 , the bounded operator $T_{C C^{\prime}}^{*}$ is well-defined. It remains to show that By definition, for each $f \in \mathcal{H}$, we have

$$
A_{C C^{\prime}}\left\|K^{*} f\right\|^{2} \leq \sum_{i \in I} w_{i}^{2}<\pi_{W_{i}} C f, \pi_{W_{i}} C^{\prime} f>\leq B_{C C^{\prime}}\|f\|^{2} .
$$

In particular, we have

$$
A_{C C^{\prime}}\left\|K^{*} f\right\|^{2} \leq\left\langle S_{C C^{\prime}} f, f\right\rangle \leq\left\|S_{C C^{\prime}} f\right\|\|f\| .
$$

Since, $\quad S_{C C^{\prime}}=T^{*} T$, then

$$
\left\|S_{C C^{\prime}} f\right\|\|f\| \leq\|T\|\|T f\|\|f\| .
$$

Hence,

$$
A_{C C^{\prime}}\|T\|^{-1}\left\|K^{*} f\right\|^{2} \leq\|T f\|\|f\| .
$$

Since $\mathcal{R}_{K}$ is closed, and via lemma we have

$$
\left.K K^{\dagger}\right|_{\mathcal{R}_{K}}=i d_{\mathcal{R}_{K}}
$$

so we have

$$
i d_{\mathcal{R}_{K}}^{*}=\left(\left.K^{\dagger}\right|_{\mathcal{R}_{K}}\right)^{*} K^{*} .
$$


Hence for any $f \in \mathcal{R}_{K}$

$$
\|f\|=\left\|\left(\left.K^{\dagger}\right|_{\mathcal{R}_{K}}\right)^{*} K^{*} f\right\|,
$$

that is,

$$
\|f\|^{2} \leq\left\|\left(K^{*}\right)^{\dagger}\right\|^{2}\left\|K^{*} f\right\|^{2}
$$

Therefore, we have

$$
A_{C C^{\prime}}\|f\|^{2} \leq A_{C C^{\prime}}\left\|\left(K^{*}\right)^{\dagger}\right\|^{2}\left\|K^{*} f\right\|^{2} .
$$

Hence,

$$
\begin{gathered}
\frac{A_{C C^{\prime}}}{\left\|\left(K^{*}\right)^{\dagger}\right\|^{2}}\|f\|^{2} \leq A_{C C^{\prime}}\left\|K^{*} f\right\|^{2} . \\
\|T f\| \geq \frac{A_{C C^{\prime}}}{\|T\|\left\|\left(K^{*}\right)^{\dagger}\right\|^{2}}\|f\| .
\end{gathered}
$$

Thus, $T_{C C^{\prime}}^{*}$ is a surjective operator.

Conversely, let $T_{C C^{\prime}}^{*}$ be a well-defined, bounded and surjective, then theorem 9 shows that $\mathcal{W}$ is a $\left(C, C^{\prime}\right)$-controlled Bessel $K$-fusion sequence for $\mathcal{H}$. Therefore, for each $f \in \mathcal{H}$, since $T_{C C^{\prime}}^{*}$ is surjective, then, by Lemma 2 , there exists an operator $\left(T_{C C^{\prime}}^{*}\right)^{\dagger}: \mathcal{H} \longrightarrow \mathcal{K}$, such that

$$
T_{C C^{\prime}}^{*}\left(T_{C C^{\prime}}^{*}\right)^{\dagger}=i d
$$

Hence,

$$
T_{C C^{\prime}}^{\dagger} T_{C C^{\prime}}=i d
$$

So, for each $f \in \mathcal{H}$, we have

$$
\begin{aligned}
\left\|K^{*} f\right\|^{2} & \leq\|K\|^{2}\left\|T_{C C^{\prime}}^{\dagger}\right\|^{2}\left\|T_{C C^{\prime}} f\right\|^{2} \\
& =\left\|T_{C C^{\prime}}^{+}\right\|^{2}\|K\|^{2} \sum_{i \in I} w_{i}^{2}<\pi_{W_{i}} C f, \pi_{W_{i}} C^{\prime} f>.
\end{aligned}
$$

Therefore, $\mathcal{W}$ is a $\left(C, C^{\prime}\right)$-controlled $K$-fusion frame for $\mathcal{H}$.

Proposition 11. Let $K \in B(\mathcal{H}), C, C^{\prime} \in G L^{+}(\mathcal{H})$ and let $\mathcal{W}$ be a $\left(C, C^{\prime}\right)$-controlled $K$-fusion frame for $\mathcal{H}$ with bounds $A_{C C^{\prime}}$ and $B_{C C^{\prime}}$ with $\overline{\mathcal{R}}_{T^{*}}$ is orthogonally complemented. If $T \in B(\mathcal{H})$ with $\mathcal{R}_{T} \subset \mathcal{R}_{K}$. Then $\mathcal{W}$ is a $\left(C, C^{\prime}\right)$-controlled $T$-fusion frame for $\mathcal{H}$.

Proof. Assume that $\mathcal{W}$ be a $\left(C, C^{\prime}\right)$-controlled $K$-fusion frame for $\mathcal{H}$ with bounds $A_{C C^{\prime}}$ and $B_{C C^{\prime}}$. Then for each $f \in \mathcal{H}$, we have

$$
A_{C C^{\prime}}\left\langle K^{*} f, K^{*} f\right\rangle \leq \sum_{i \in I} w_{i}^{2}\left\langle\pi_{W_{i}} C f, \pi_{W_{i}} C^{\prime} f\right\rangle \leq B_{C C^{\prime}}\langle f, f\rangle .
$$

Since $\mathcal{R}_{T} \subset \mathcal{R}_{K}$, so by using lemma 4 , there exists some $\lambda>0$ such that

$$
T T^{*} \leq \lambda K K^{*} .
$$

This implies that for all $f \in \mathcal{H}$, we have

$$
A_{C C^{\prime}}\left\langle T^{*} f, T^{*} f\right\rangle \leq A_{C C^{\prime}} \lambda\left\langle K^{*} f, K^{*} f\right\rangle .
$$


Therfore,

$$
\frac{A_{C C^{\prime}}}{\lambda}\left\langle T^{*} f, T^{*} f\right\rangle \leq A_{C C^{\prime}}\left\langle K^{*} f, K^{*} f\right\rangle \leq \sum_{i \in I} w_{i}^{2}\left\langle\pi_{W_{i}} C f, \pi_{W_{i}} C^{\prime} f\right\rangle \leq B_{C C^{\prime}}\langle f, f\rangle .
$$

Then, $\mathcal{W}$ is a $\left(C, C^{\prime}\right)$-controlled $T$-fusion frame for $\mathcal{H}$ with bounds $\frac{A_{C C^{\prime}}}{\lambda}$ and $B_{C C^{\prime}}$.

Theorem 12. Let $K_{1}, K_{2} \in B(\mathcal{H})$ such that $\mathcal{R}_{K_{1}^{*}} \perp \mathcal{R}_{K_{2}^{*}}$. If $\mathcal{W}$ is a $\left(C, C^{\prime}\right)$-controlled $K_{i}$-fusion frame for $\mathcal{H}(i=1,2)$. Then $\mathcal{W}$ is a $\left(C, C^{\prime}\right)$-controlled $\left(\alpha K_{1}+\beta K_{2}\right)$-fusion frame for $\mathcal{H}$, where $\alpha, \beta \in \mathbb{C}$.

Proof. Since $\mathcal{W}$ is a $\left(C, C^{\prime}\right)$-controlled $K_{i}$-fusion frame for $\mathcal{H}(i=1,2)$, there exist $A_{C C^{\prime}}^{j}, B_{C C^{\prime}}^{j}>$ 0 , such that for all $f \in \mathcal{H}, j=1,2$, we have

$$
A_{C C^{\prime}}^{j}\left\langle K_{j}^{*} f, K_{j}^{*} f\right\rangle \leq \sum_{i \in I} w_{i}^{2}\left\langle\pi_{W_{i}} C f, \pi_{W_{i}} C^{\prime} f\right\rangle \leq B_{C C^{\prime}}^{j}\langle f, f\rangle .
$$

Then for any $f \in \mathcal{H}$, we have

$$
\begin{aligned}
& \left\langle\left(\alpha K_{1}+\beta K_{2}\right)^{*} f,\left(\alpha K_{1}+\beta K_{2}\right)^{*} f\right\rangle=\left\langle\bar{\alpha} K_{1}^{*} f+\bar{\beta} K_{2}^{*} f, \bar{\alpha} K_{1}^{*} f+\bar{\beta} K_{2}^{*} f\right\rangle \\
= & |\alpha|^{2}\left\langle K_{1}^{*} f, K_{1}^{*} f\right\rangle+\bar{\alpha} \beta\left\langle K_{1}^{*} f, K_{2}^{*} f\right\rangle+\alpha \bar{\beta}\left\langle K_{2}^{*} f, K_{1}^{*} f\right\rangle+|\beta|^{2}\left\langle K_{2}^{*} f, K_{2}^{*} f\right\rangle .
\end{aligned}
$$

Since $\mathcal{R}_{K_{1}} \perp \mathcal{R}_{K_{2}}$, then, for any $f \in \mathcal{H}$, we have

$$
\begin{aligned}
& \left\langle K_{1}^{*} f, K_{2}^{*} f\right\rangle=0, \\
& \left\langle K_{2}^{*} f, K_{1}^{*} f\right\rangle=0 .
\end{aligned}
$$

Thus,

$$
\left\langle\left(\alpha K_{1}+\beta K_{2}\right)^{*} f,\left(\alpha K_{1}+\beta K_{2}\right)^{*} f\right\rangle=|\alpha|^{2}\left\langle K_{1}^{*} f, K_{1}^{*} f\right\rangle+|\beta|^{2}\left\langle K_{2}^{*} f, K_{2}^{*} f\right\rangle .
$$

Therfore, for any $f \in \mathcal{H}$, we have

$$
\begin{aligned}
|\alpha|^{2}\left\langle K_{1}^{*} f, K_{1}^{*} f\right\rangle+|\beta|^{2}\left\langle K_{2}^{*} f, K_{2}^{*} f\right\rangle & \leq\left(\frac{|\alpha|^{2}}{A_{C C^{\prime}}^{1}}+\frac{|\beta|^{2}}{A_{C C^{\prime}}^{2}}\right) \sum_{i \in I} w_{i}^{2}\left\langle\pi_{W_{i}} C f, \pi_{W_{i}} C^{\prime} f\right\rangle \\
& \left.\leq \frac{\left(A_{C C^{\prime}}^{2}|\alpha|^{2}+A_{C C^{\prime}}^{1}|\beta|^{2}+1\right)}{A_{C C^{\prime}}^{1} A_{C C^{\prime}}^{2}}\right) \sum_{i \in I} w_{i}^{2}\left\langle\pi_{W_{i}} C f, \pi_{W_{i}} C^{\prime} f\right\rangle .
\end{aligned}
$$

Then,

$$
\begin{aligned}
0 & <\frac{A_{C C^{\prime}}^{1} A_{C C^{\prime}}^{2}}{\left(A_{C C^{\prime}}^{2}|\alpha|^{2}+A_{C C^{\prime}}^{1}|\beta|^{2}+1\right)}\left(|\alpha|^{2}\left\langle K_{1}^{*} f, K_{1}^{*} f\right\rangle+|\beta|^{2}\left\langle K_{2}^{*} f, K_{2}^{*} f\right\rangle\right) \\
& \leq \sum_{i \in I} w_{i}^{2}\left\langle\pi_{W_{i}} C f, \pi_{W_{i}} C^{\prime} f\right\rangle .
\end{aligned}
$$

Then, we have

$$
\frac{A_{C C^{\prime}}^{1} A_{C C^{\prime}}^{2}\left\langle\left(\alpha K_{1}+\beta K_{2}\right)^{*} f,\left(\alpha K_{1}+\beta K_{2}\right)^{*} f\right\rangle}{\left(A_{C C^{\prime}}^{2}|\alpha|^{2}+A_{C C^{\prime}}^{1}|\beta|^{2}+1\right)} \leq \sum_{i \in I} w_{i}^{2}\left\langle\pi_{W_{i}} C f, \pi_{W_{i}} C^{\prime} f\right\rangle,
$$


then,

$$
\begin{aligned}
\sum_{i \in I} w_{i}^{2}\left\langle\pi_{W_{i}} C f, \pi_{W_{i}} C^{\prime}\right\rangle & =\frac{1}{2}\left(\sum_{i \in I} w_{i}^{2}\left\langle\pi_{W_{i}} C f, \pi_{W_{i}} C^{\prime} f\right\rangle+\sum_{i \in I} w_{i}^{2}\left\langle\pi_{W_{i}} C f, \pi_{W_{i}} C^{\prime} f\right\rangle\right) \\
& \leq \frac{B_{C C^{\prime}}^{1}+B_{C C^{\prime}}^{2}}{2}\langle f, f\rangle .
\end{aligned}
$$

Thus, $\mathcal{W}$ is a $\left(C, C^{\prime}\right)$-controlled $\alpha K_{1}+\beta K_{2}$-fusion frame with bounds $\frac{A_{C C^{\prime}}^{1} A_{C C^{\prime}}^{2}}{\left(|\alpha|^{2} A_{C C^{\prime}}^{2}+|\beta|^{2} A_{C C^{\prime}}^{1}+1\right)}$ and $\frac{B_{C C^{\prime}}^{1}+B_{C C^{\prime}}^{2}}{2}$.

Lemma 13. Let $K \in B(\mathcal{H})$, and $C, C^{\prime} \in G L^{+}(\mathcal{H})$ such that $C C^{\prime}=C^{\prime} C$. Assume that $C K=K C, C^{\prime} K=K C^{\prime} ; S C=C S, S C^{\prime}=C^{\prime} S$ and $\pi_{W_{i}} C=C \pi_{W_{i}}, \pi_{W_{i}} C^{\prime}=C^{\prime} \pi_{W_{i}}$. Then, $\mathcal{W}$ is a $\left(C, C^{\prime}\right)$-controlled $K$-fusion frame for $\mathcal{H}$ if and only if $\mathcal{W}$ is a K-fusion frame for $\mathcal{H}$.

Where $S$ is the K-fusion frame operator (2.3), defined by

$$
S f=\sum_{i \in I} w_{i}^{2} \pi_{W_{i}} f, \quad f \in \mathcal{H} .
$$

Proof. Assume that $\mathcal{W}$ is a $K$-fusion frame with bounds $A$ and $B$. Then for each $f \in \mathcal{H}$, we have

$$
A\left\|K^{*} f\right\|^{2} \leq \sum_{i \in I} w_{i}^{2}\left\|\pi_{W_{i}} f\right\|^{2} \leq B\|f\|^{2} .
$$

Since, $C$ and $C^{\prime}$ are linear bounded operators, applying 2.1, there exist constants $m, m^{\prime}, M$ and $M^{\prime}>0$ such that

$$
\begin{aligned}
& \left\{\begin{array}{c}
m I \leq C \leq M I \\
m^{\prime} I \leq C^{\prime} \leq M^{\prime} I .
\end{array}\right. \\
& \langle S C f, f\rangle=\langle f, C S f\rangle .
\end{aligned}
$$

Then,

$$
m K K^{*} \leq C S \leq M S \leq M B I
$$

We deduce that

$$
m m^{\prime} K K^{*} \leq C^{\prime} S C \leq M M^{\prime} B I .
$$

Therfore, for each $f \in \mathcal{H}$, we have

$$
m m^{\prime} A\left\langle K^{*} f, K^{*} f\right\rangle \leq \sum_{i \in I} w_{i}^{2}\left\langle\pi_{W_{i}} C f \pi_{W_{i}} C^{\prime} f\right\rangle \leq M M^{\prime} B\|f\|^{2} .
$$

Thus, $\mathcal{W}$ is a $\left(C, C^{\prime}\right)$-controlled $K$-fusion frame.

Conversely, Assume that $\mathcal{W}$ is a $\left(C, C^{\prime}\right)$-controlled $K$-fusion frame with bounds $A$ and $B$. Then for each $f \in \mathcal{H}$, we have

$$
A_{C C^{\prime}}\left\|K^{*} f\right\|^{2} \leq \sum_{i \in I} w_{i}^{2}\left\langle\pi_{W_{i}} C f, \pi_{W_{i}} C^{\prime} f \leq B_{C C^{\prime}}\|f\|^{2} .\right.
$$


By assumption, $C, C^{\prime} \in G L^{+}(\mathcal{H}), C C^{\prime}=C^{\prime} C$ and via theorem 5, we have

$$
\begin{aligned}
\left((C)^{\frac{1}{2}}\left(C^{\prime}\right)^{-\frac{1}{2}}\right)^{*} & =\left(C^{* *}\right)^{-\frac{1}{2}}\left(C^{*}\right)^{\frac{1}{2}} \\
& =\left(C^{\prime}\right)^{-\frac{1}{2}}(C)^{\frac{1}{2}} \\
& =(C)^{\frac{1}{2}}\left(C^{\prime}\right)^{-\frac{1}{2}}
\end{aligned}
$$

Then, for each $f \in \mathcal{H}$, we have

$$
\begin{aligned}
& A_{C C^{\prime}}\left\langle K^{*} f, K^{*} f\right\rangle=A_{C C^{\prime}}\left\langle\left(C C^{\prime}\right)^{\frac{1}{2}}\left(C C^{\prime}\right)^{-\frac{1}{2}} K^{*} f,\left(C C^{\prime}\right)^{\frac{1}{2}}\left(C C^{\prime}\right)^{-\frac{1}{2}} K^{*} f\right\rangle \\
& \leq\left\|\left(C C^{\prime}\right)^{\frac{1}{2}}\right\|^{2} \sum_{i \in I} w_{i}^{2}\left\langle\pi_{W_{i}} C\left(C C^{\prime}\right)^{-\frac{1}{2}} f, \pi_{W_{i}} C^{\prime}\left(C C^{\prime}\right)^{-\frac{1}{2}} f\right\rangle \\
&=\left\|\left(C C^{\prime}\right)^{\frac{1}{2}}\right\|^{2} \sum_{i \in I} w_{i}^{2}\left\langle\pi_{W_{i}}(C)^{\frac{1}{2}}\left(C^{\prime}\right)^{-\frac{1}{2}} f, \pi_{W_{i}}\left(C^{\prime}\right)^{\frac{1}{2}}(C)^{-\frac{1}{2}} f\right\rangle \\
&=\left\|\left(C C^{\prime}\right)^{\frac{1}{2}}\right\|^{2} \sum_{i \in I} w_{i}^{2}\left\langle\left(\left(C^{\prime}\right)^{\frac{1}{2}}(C)^{-\frac{1}{2}}\right)^{*} \pi_{W_{i}}(C)^{\frac{1}{2}}\left(C^{\prime}\right)^{-\frac{1}{2}} f, f\right\rangle \\
&=\left\|\left(C C^{\prime}\right)^{\frac{1}{2}}\right\|^{2} \sum_{i \in I} w_{i}^{2}\left\langle\left(\left(C^{\prime}\right)^{\frac{1}{2}}(C)^{-\frac{1}{2}}\right)^{*} \pi_{W_{i}}(C)^{\frac{1}{2}}\left(C^{\prime}\right)^{-\frac{1}{2}} f, f\right\rangle \\
&=\left\|\left(C C^{\prime}\right)^{\frac{1}{2}}\right\|^{2} \sum_{i \in I} w_{i}^{2}\left\langle\pi_{W_{i}}\left(C^{\prime}\right)^{\frac{1}{2}}(C)^{-\frac{1}{2}}(C)^{\frac{1}{2}}\left(C^{\prime}\right)^{-\frac{1}{2}} f, f\right\rangle \\
&=\left\|\left(C C^{\prime}\right)^{\frac{1}{2}}\right\|^{2}\left\langle\sum_{i \in I} w_{i}^{2} \pi_{W_{i}} f, f\right\rangle . \\
& \Longrightarrow \quad A_{C C^{\prime}}\left\|\left(C C^{\prime}\right)^{\frac{1}{2}}\right\|^{-2}\left\langle K^{*} f, K^{*} f\right\rangle \leq \sum_{i \in I} w_{i}^{2}\left\langle\pi_{W_{i}} f, \pi_{W_{i}} f\right\rangle .
\end{aligned}
$$

In the other hand

$$
\sum_{i \in I} w_{i}^{2}\left\langle\pi_{W_{i}} f, \pi_{W_{i}} f\right\rangle=\langle S f, f\rangle,
$$

where $\quad S f=\sum_{i \in I} w_{i}^{2} \pi_{W_{i}} f$.

$$
\begin{aligned}
\langle S f, f\rangle & =\left\langle\left(C C^{\prime}\right)^{-\frac{1}{2}}\left(C C^{\prime}\right)^{\frac{1}{2}} S f, f\right\rangle \\
& =\left\langle\left(C C^{\prime}\right)^{\frac{1}{2}} S f,\left(\left(C C^{\prime}\right)^{-\frac{1}{2}}\right)^{*} f\right\rangle \\
& =\left\langle\left(C C^{\prime}\right)^{\frac{1}{2}} S f,\left(C C^{\prime}\right)^{-\frac{1}{2}} f\right\rangle \\
& =\left\langle C C^{\prime}\left(C C^{\prime}\right)^{-\frac{1}{2}} S f,\left(C C^{\prime}\right)^{-\frac{1}{2}} f\right\rangle \\
& =\left\langle C^{\prime} S C\left(C C^{\prime}\right)^{-\frac{1}{2}} f,\left(C C^{\prime}\right)^{-\frac{1}{2}} f\right\rangle \\
& \leq B_{C C^{\prime}}\left\|\left(C C^{\prime}\right)^{-\frac{1}{2}}\right\|^{2}\|f\|^{2} .
\end{aligned}
$$

Thus, $\mathcal{W}$ is a $K$-fusion frame with bounds $A_{C C^{\prime}}\left\|\left(C C^{\prime}\right)^{\frac{1}{2}}\right\|^{-2}$ and $B_{C C^{\prime}}\left\|\left(C C^{\prime}\right)^{-\frac{1}{2}}\right\|^{2}$. 
Theorem 14. Let $K \in B(\mathcal{H})$, let $\mathcal{W}$ be a $(C, C)$-controlled $K$-fusion frame with bounds $A_{C C}$ and $B_{C C}$. If $U \in \mathcal{B}(\mathcal{H})$ is an invertible operator such that $U^{*} C=C U^{*}$ and $K^{*}\left(U^{*}\right)^{-1}=\left(U^{*}\right)^{-1} K^{*}$, then $\left(U W_{i}, w_{i}\right)_{i \in I}$ is a $(C, C)$-controlled $\mathrm{K}$-fusion frame for $\mathcal{H}$.

Proof. Assume that $\mathcal{W}$ is a $(C, C)$-controlled $K$-fusion frame with bounds $A_{C C}$ and $B_{C C}$. By definition, for each $f \in \mathcal{H}$, we have

$$
A_{C C}\left\|K^{*} f\right\|^{2} \leq \sum_{i \in I} w_{i}^{2}\left\|\pi_{W_{i}} C f\right\|^{2} \leq B_{C C}\|f\|^{2} .
$$

Now, let $f \in \mathcal{H}$. Via lemma 3 and since $U W_{i}$ is closed, we have

$$
\begin{aligned}
\left\|\pi_{W_{i}} C U^{*} f\right\|=\left\|\pi_{W_{i}} U^{*} C f\right\|=\left\|\pi_{W_{i}} U^{*} \pi_{\overline{U W_{i}}} C f\right\| & =\left\|\pi_{W_{i}} U^{*} \pi_{U W_{i}} C f\right\| \\
& \leq\|U\|\left\|\pi_{U W_{i}} C f\right\| .
\end{aligned}
$$

Therefore,

$$
\begin{aligned}
& A_{C C}\left\|K^{*} U^{*} f\right\|^{2} \leq \sum_{i \in I} w_{i}^{2}\left\|\pi_{W_{i}} C U^{*} f\right\|^{2} \\
& \leq\|U\|^{2} \sum_{i \in I} w_{i}^{2}\left\|\pi_{U W_{i}} C f\right\|^{2} . \\
&\left\|K^{*} f\right\|^{2}=\left\|K^{*}\left(U^{*}\right)^{-1} U^{*} f\right\|^{2}=\left\|\left(U^{*}\right)^{-1} K^{*} U^{*} f\right\|^{2} \\
& \leq\left\|U^{-1}\right\|^{2}\left\|K^{*} U^{*} f\right\|^{2} .
\end{aligned}
$$

Then, we have

$$
\frac{A_{C C}}{\left\|U^{-1}\right\|^{-2}\|U\|^{-2}}\left\|K^{*} f\right\|^{2} \leq \sum_{i \in I} w_{i}^{2}\left\|\pi_{U W_{i}} C f\right\|^{2} .
$$

On the other hand, via lemma 3, we obtain with $U^{-1}$ instead of $T$ :

$$
\pi_{U W_{i}}=\pi_{U W_{i}}\left(U^{*}\right)^{-1} \pi_{W_{i}} U^{*} .
$$

Thus,

$$
\begin{aligned}
\left\|\pi_{U W_{i}} C f\right\|^{2} & =\left\|\pi_{U W_{i}}\left(U^{*}\right)^{-1} \pi_{W_{i}} U^{*} C f\right\|^{2} \\
& \leq\left\|U^{-1}\right\|^{2}\left\|\pi_{W_{i}} U^{*} C f\right\|^{2}
\end{aligned}
$$

and it follows

$$
\sum_{i \in I} w_{i}^{2}\left\|\pi_{U W_{i}} C f\right\|^{2} \leq\left\|U^{-1}\right\|^{2} \sum_{i \in I} w_{i}^{2}\left\|\pi_{W_{i}} U^{*} C f\right\|^{2}
$$

hence,

$$
\sum_{i \in I} w_{i}^{2}\left\|\pi_{U W_{i}} C f\right\|^{2} \leq B_{C C}\left\|U^{-1}\right\|^{2}\|U\|^{2}\|f\|^{2} .
$$

Thus, $\mathcal{W}$ is a $(C, C)$-controlled $K$-fusion frame with bounds $A_{C C}\left\|U^{-1}\right\|^{-2}\|U\|^{-2}$ and $B_{C C}\left\|U^{-1}\right\|^{2}\|U\|^{2}$ 
Corollary 15. Let $K \in B(\mathcal{H})$, let $\mathcal{W}$ be a $(C, C)$-controlled $K$-fusion frame with bounds $A_{C C}$ and $B_{C C}$ If $U \in \mathcal{B}(\mathcal{H})$ is a unitary operator such that $U^{-1} C=C U^{-1}$ and $K^{*} U=U K^{*}$, then $\left(U W_{i}, w_{i}\right)_{i \in I}$ is a $(C, C)$-controlled $K$-fusion frame for $\mathcal{H}$.

Proof. The result follows from Theorem 14.

\section{Perturbation on Controlled $K$-fusion frame}

The following result provides a sufficient condition on a family of closed subspaces of $\mathcal{H}$ to be a controlled $K$-fusion frame, in the presence of another controlled $K$-fusion frame. In fact it is a generalisation of Proposition 2.4 in [2], Proposition 4.6 in [8] and Proposition 2.6 in [17].

Proposition 16. Let $K \in B(\mathcal{H})$ be a closed range operator $\mathcal{R}_{K}$, let $T, U \in G L(\mathcal{H})$ and let $\mathcal{W}=$ $\left\{W_{i}, w_{i}\right\}_{i \in I}$ be a $\left(C, C^{\prime}\right)$-controlled $K$-fusion frame for $\mathcal{H}$ with lower and upper bounds $A_{C C^{\prime}}$ and $B_{C C^{\prime}}$, respectively. Let $\left\{V_{i}\right\}_{i \in I}$ be a family of closed subspaces of $\mathcal{H}$. If there exists a number $0<R<A_{C C^{\prime}}$ such that

$$
0<\sum_{i \in I} w_{i}^{2}\left\langle C^{*}\left(\pi_{V_{i}}-\pi_{W_{i}}\right) C f, f\right\rangle \leq R\|f\|^{2}, \forall f \in \mathcal{H},
$$

then $\mathcal{V}=\left\{V_{i}, w_{i}\right\}_{i \in I}$ is a $\left(C, C^{\prime}\right)$-controlled Bessel $K$-fusion sequence for $\mathcal{H}$ and $a\left(C, C^{\prime}\right)$-controlled K-fusion frame for $\mathcal{R}_{K}$.

Proof. Let $f \in \mathcal{H}$. Considering that the family $\mathcal{W}=\left\{W_{i}, w_{i}\right\}_{i \in I}$ is a $\left(C, C^{\prime}\right)$-controlled $K$-fusion frame for $\mathcal{H}$, we have

$$
A_{C C^{\prime}}\left\|K^{*} f\right\|^{2} \leq \sum_{i \in I} w_{i}^{2}\left\langle C^{\prime *} \pi_{W_{i}} C f, f\right\rangle \leq B_{C C^{\prime}}\|f\|^{2} .
$$

Firstly, let us prove that $\left\{V_{i}, w_{i}\right\}_{i \in I}$ is a $\left(C, C^{\prime}\right)$-controlled Bessel $K$-fusion sequence for $\mathcal{H}$. We have

$$
\begin{aligned}
\sum_{i \in I} w_{i}^{2}\left\langle C^{*} \pi_{V_{i}} C f, f\right\rangle & =\sum_{i \in I} w_{i}^{2}\left\langle C^{\prime *}\left(\pi_{V_{i}}-\pi_{W_{i}}\right) C f, f\right\rangle+\sum_{i \in I} w_{i}^{2}\left\langle C^{*} \pi_{W_{i}} C f, f\right\rangle \\
& \leq R\|f\|^{2}+B_{C C^{\prime}}\|f\|^{2}
\end{aligned}
$$

consequently,

$$
\sum_{i \in I} w_{i}^{2}\left\langle C^{\prime *} \pi_{V_{i}} C f, f\right\rangle \leq\left(R+B_{C C^{\prime}}\right)\|f\|^{2} .
$$

Now, let us establish for $\left\{V_{i}, w_{i}\right\}_{i \in I}$ the left-hand side. We obtain

$$
\begin{gathered}
\sum_{i \in I} w_{i}^{2}\left\langle C^{*} \pi_{V_{i}} C f, f\right\rangle=\sum_{i \in I} w_{i}^{2}\left\langle C^{*} \pi_{W_{i}} C f, f\right\rangle+\sum_{i \in I} w_{i}^{2}\left\langle C^{*}\left(\pi_{V_{i}}-\pi_{W_{i}}\right) C f, f\right\rangle \\
\geq \sum_{i \in I} w_{i}^{2}\left\langle C^{*} \pi_{W_{i}} C f, f\right\rangle-\sum_{i \in I} w_{i}^{2}\left\langle C^{*}\left(\pi_{V_{i}}-\pi_{W_{i}}\right) C f, f\right\rangle \\
\geq A_{C C^{\prime}}\left\|K^{*} f\right\|^{2}-R\|f\|^{2} .
\end{gathered}
$$


Therfore, for any $f \in \mathcal{R}_{K}$, we have

$$
\|f\|=\left\|\left(\left.K^{\dagger}\right|_{\mathcal{R}_{K}}\right)^{*} K^{*} f\right\| \leq\left\|K^{\dagger}\right\|\left\|K^{*} f\right\|,
$$

that is, $\left\|K^{*} f\right\|^{2} \geq\left\|K^{\dagger}\right\|^{-2}\|\| f \|^{2}$.

$$
-R\|f\|^{2} \geq-R\left\|K^{\dagger}\right\|^{-2}\left\|K^{*} f\right\|^{2}
$$

Then, according to (4.2) and (4.3), we have

$$
\sum_{i \in I} w_{i}^{2}\left\langle C^{*} \pi_{V_{i}} C f, f\right\rangle \geq\left(A_{C C^{\prime}}-R\left\|K^{\dagger}\right\|^{-2}\right)\left\|K^{*} f\right\|^{2} .
$$

Which completes the proof.

Aknowlegements : The authors would like to thank the referee for carefully reading our manuscript and for giving such constructive comments which substantially helped improving the quality of the paper.

\section{References}

[1] P. Balaz, J-P. Antoine and A.Grybos, Wighted and controlled frames. Int. J. Wavelets Multi. Inf. Process., 8(10) (2010) 109-132.

[2] M. S. Asgari, A. Khosravi, Frames and bases of subspaces in Hilbert spaces, J. Math. Anal. appl. 308 (2005),541-553.

[3] F. Arabyani-Neyshaburi and A. Arefijamaal. Characterization and construction of K-fusion frames and their duals in Hilbert spaces. Results Math, 73 (2018).

[4] A. Arefijamaal, F. Arabyani-Neyshaburi, Some properties of alternate duals and ap-proximate alternate duals of fusion frames. Turk J. Math (2017) 41: 119112033.

[5] L. Arambasic, On frames for countably generated Hilbert $C^{*}$-modules, Proc. Amer. Math. Soc. 135(2007) 469-478.

[6] P. G. Casazza, G. Kutyniok, Frames and subspaces. In: Wavelets, Frames, and Operator Theory. Contemporary Mathematics, vol. 345, pp. 87113. American Mathematical Society, Providence (2004).

[7] O. Christensen, An introduction to frames and Riesz bases, Birkhauser, Boston (2016).

[8] O. Christensen, C. Heil, Perturbations of Banach frames and atomic decomposition, Math. Nachr. 185(1997), 33-47.

[9] P. G. Casazza, G. Kutyniok and S. Li, Fusion frames and distributed processing, Appl. Comput. Harmon. Anal., 25 (2008), 114-132.

[10] I. Daubechies, A. Grossmann, Y. Meyer, Painless non orthogonal expansions, J. Math. Phys. 27(1986) 1271-1283.

[11] R. J. Duffin and A. C. Schaeffer, A class of nonharmonic Fourier series, Trans. Am. Math. Soc 72. (1952), 341366.

[12] X.Fang, J. Yu and H. Yao, Solutions to operator equations On Hilbert C-modules, linear Alg. Appl, 431(11) (2009) 2142-2153.

[13] I. ICHIM , Sur la racine carre d'un oprateur linare, Bulletin mathmatique, Vol. 29 (77), No. 1 (1985), pp. 45-54 (10 pages).

[14] L. Gavruta, On the duality of fusion frames, J. Math. Anal. Appl. 333 (2007), 871-879.

[15] L. Gavruta, Frames for operators, Appl. Comput. Harmon. Anal. 32 (2012), 139-144.

[16] G. Hong, P.Li, fusion frames for Hilbert spaces. J. Inequal Appl 2019, 226 (2019).

[17] A. Khosravi and K. Musazadeh, Controlled fusion frames, Methods Funct. Anal. Topology (2012), 18(3), 256265.

[18] Ai Fang Liu , Peng Tong Li,K-fusion Frames and the Corresponding Generators for Unitary Systems. Acta. Math. Sin.-English Ser. 34, 843854(2018).

[19] D. Han and D. R. Larson, Frames, bases and group representations, Memoirs of the American Mathematical Society, vol. 147, no. 697, pp. 191, 2000. 
[20] M. Nouri, A. Rahimi and Sh. Najafzadeh,Controlled K-frames in Hilbert Spaces,J. of Ramanujan Society of Math. and Math. Sc.,4(2)(2015)39-50.

[21] W. Paschke, Inner product modules over $B^{*}$-algebras, Trans. Amer. Math. Soc., (182)(1973), 443-468.

[22] Sun, W., G-Frames and G-Riesz bases. J. Math. Anal. Appl.326 , 437-452 (2006).

[23] R. Young, An Introduction to Nonharmonic Fourier Series, Academic Press, New York, NY, USA, 1980. Applied and Numerical Harmonic Analysis, Birkhauser, Boston,Mass, USA, 2003. 